\title{
CERAMIC SONOTRODES FOR LIGHT ALLOY MELT TREATMENT
}

\author{
H. Puga $(1)$ and S. D. Tohidi \\ CMEMS - UMinho, Department of Mechanical Engineering, University of Minho, Guimarães, Portugal \\ V. H. Carneiro and J. Meireles \\ METRICS - UMinho, Department of Mechanical Engineering, University of Minho, Guimarães, Portugal
}

M. Prokic

MPI Interconsulting, Le Locle, Switzerland

Copyright (C) 2020 American Foundry Society

https://doi.org/10.1007/s40962-020-00476-5

\begin{abstract}
Alloy melt treatment by ultrasonic vibration is a physical processing technique that has been gathering the support of the scientific community. The use of metallic sonotrodes for this purpose has been proven very efficient; however, it promotes melt inclusion by sonotrode erosion. Such an issue is being addressed by the use of ceramic sonotrodes in low-amplitude resonance. Given that these novel sonotrodes generally have complex shapes and low displacements, this study shows an innovative approach for their

radial resonance in resonant ceramic sonotrodes. Velocity time-domain profiles are shown to be dependent on the position, and even though the radial natural frequencies of ceramic sonotrodes have low amplitudes, they are proposed as an efficient tool for melt treatment. While characterizing the radial natural mode in ceramic sonotrodes, this study proves that their low-amplitude Lamb waves are responsible for the refinement of $\alpha$-grains and secondary phases in light alloys.
\end{abstract} characterization. Based on scanning laser Doppler vibrometry, the signal processing Python-based script was used to map the overall resonant behavior of a tubular SiAlON sonotrode, and this route is able to characterize the complex shapes in low-amplitude and high-frequency
Keywords: ceramic sonotrode, ultrasonic transducer, lamb waves, Grain refinement, casting

\section{Introduction}

In recent years, there has been an increasing interest in ultrasonic tools. While high-frequency excitations were not usually considered relevant due to low amplitudes, current studies show that they may be extremely beneficial in many practical applications, from medical, ${ }^{1}$ food $^{2}$ to manufacturing industries. ${ }^{3}$ It has been reported that ultrasounds are useful in a fluid medium for uses such as chemical extraction ${ }^{4-6}$ and melt treatment. ${ }^{7-12}$ Consequently, there is extensive research on the design and characterization of ultrasonic tools. ${ }^{13-15}$

Melt treatment by ultrasonic excitation, in particular, had significant advances in the last decade. This physical processing is known to promote grain/intermetallic refinement, ${ }^{16-19}$ melt degassing ${ }^{20,21}$ and reinforcement particle dispersion, ${ }^{22-28}$ having been successfully applied to different alloys. ${ }^{29-32}$ This technique, however, has problems due to its application in high-temperatures and the use of metallic sonotrodes that may have an affinity to react with the melts. It is known that prolonged uses of metallic sonotrodes may cause erosion and promote inclusions in the melt. ${ }^{33-35}$ This issue is being addressed by the use of ceramic-based sonotrodes. While the vibration of ceramic resonating tools may not initially seem a plausible solution, due to the low toughness of these materials, they may be safely used at high-temperature in low amplitude resonance. However, these radial high-frequency resonant modes are characterized by complex shapes and there are no specific tools/routes for their characterization. While this is essential to the design of ultrasonic tools and 
applications (e.g., melt treatment), the accuracy/precision in the measurement of these low-amplitude displacements in complex geometries is highly dependent on signal processing. Thus, the quality of signal acquisition and processing from sensors used to characterize these tools are fundamental variables for the obtaining of reliable response. There are common signal disruption issues such as superimposition of forward and backward scattered waves from structural boundaries and/or geometric irregularities, multiple vibration natural modes, wave dispersion/attenuation, changes in propagation velocity, broadband noise, among others. ${ }^{36,37}$

The steady-state waveform propagation of sonotrode is monitored at determined points in various diagonalcolumnar orders of the sonotrode using a scanning laser Doppler vibrometer at high ultrasonic frequencies ( $\sim 20 \mathrm{kHz}$ ). Given its tubular configuration and low amplitude of the displacements in radial resonance, a signal processing algorithm written in a Python-based script was used to map the overall resonant behavior. After the sonotrode characterization, the equipment was introduced in an $\mathrm{Al}-\mathrm{Si}-\mathrm{Mg}$ alloy melt to evaluate the ability of this technique to refine the $\alpha$-Al grain/intermetallic compounds and modify the eutectic Si. This is the first study that correlates the complex shape of radial resonating ceramic tubes with their ability to treat melts and refine cast products.

\section{Methodology}

\section{Ultrasonic Transducer System Setup}

Multi-frequency-mode modulated technology (MMM)generated ultrasonic energy is produced by a piezoelectric transducer (Figure 1). MMM ultrasonic vibrations are guided by an acoustic waveguide that has $35 \mathrm{~mm}$ diameter and it is $420 \mathrm{~mm}$ long, to clamps radially fixed on the sialon tube (60 mm diameter and $1200 \mathrm{~mm}$ length). The optimal operating parameters, including both frequency and phase modulation, are controlled by dedicated ultrasonic generator software. The vibration frequency and amplitude transmitted by the SiAlON tube to the liquid medium can be tuned to create a smooth steady-state liquid vibration. It is worth mentioning that, while the natural frequency of the radial eigenmode would be changed by the ratio between the diameter and length, as long as the geometry is a tube (i.e., length $\gg$ diameter $\gg$ thickness), the form of this resonant shape would be the same.

\section{Vibrometry Experimental Setup}

Scanning laser Doppler vibrometer (SLDV) was used to perform a continuous non-contact scan of the harmonic response in a SiAlON sonotrode that is excited by the ultrasonic transducer. While a Polytec OFV-512 fiber vibrometer was used to generate the laser beam and monitor the response, a VibroLaser Scanset set of mirrors and

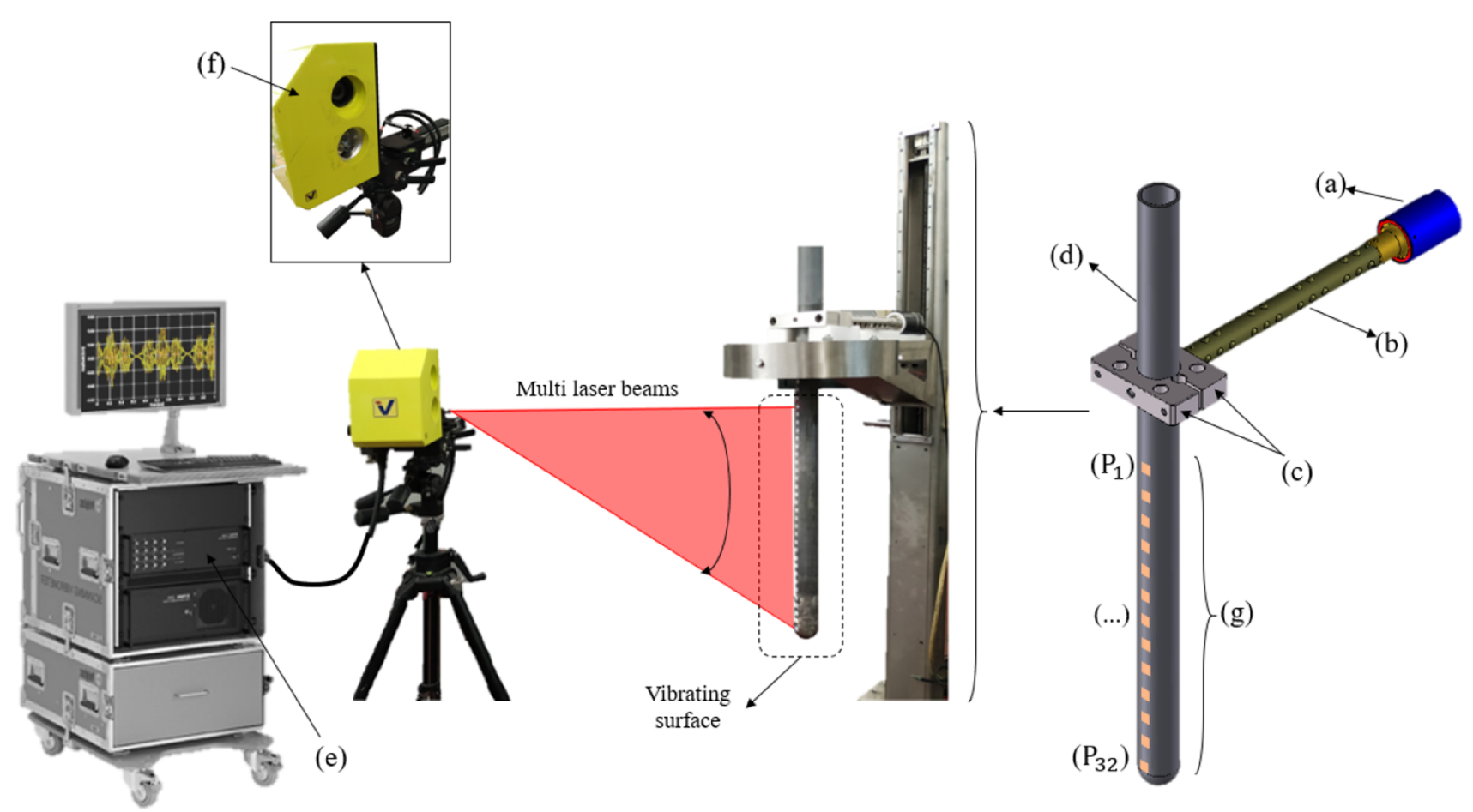

Figure 1. Experimental apparatus: (a) piezoelectric transducer; (b) waveguide; (c) clamps; (d) SiAION sonotrode; (e) fiber vibrometer; (f) mirrors/spectrum analyzer; and (g) retro-reflective tape. 
spectrum analyzer aimed the laser beam and recorded the data guaranteeing the uniformity of the process during the tests. The experimental setup of the SLDV monitors velocities during the sonotrode sonication. Retro-reflective tape was placed in a columnar line (32 measurement points, $20 \mathrm{~mm}$ apart from each other) to maximize the return light intensity. The vibrational response of each measurement point was combined to assemble a multipoint vibration analysis to plot the nodal displacement profile of the sonotrode. Signal was acquired in the form of velocity and time to estimate the frequency response function (FRF), shapes and velocities to characterize the radial response of the sialon tube.

\section{Melt Treatment and Casting}

Primary A356 (A17Si0.3Mg, Table 1) commercial ingots were sectioned into $200 \mathrm{~g}$ pieces, after being degreased, washed and dried. A SiC crucible was used to melt $5 \mathrm{~kg}$ of alloy in a resistance furnace (Figure 2), and the cruciblefurnace combination was previously heated to $300{ }^{\circ} \mathrm{C}$ for $30 \mathrm{~min}$ to reduce moisture content. Then, the furnace temperature was elevated to $700 \pm 5{ }^{\circ} \mathrm{C}$ and the alloy was inserted in the crucible. After melting, the temperature was corrected using a melt control thermocouple and kept isothermal for $30 \mathrm{~min}$ for homogenization. After this period, melt temperature was reduced to $680 \pm 5^{\circ} \mathrm{C}$, and a ceramic sonotrode was inserted $(200 \mathrm{~mm}$ insertion) to promote isothermal degassing and refinement.

The operational parameters in the ultrasonic transducer were optimized to the experimental apparatus (Figure 2a, $f=20.2 \pm 0.4 \mathrm{kHz} ; \quad$ sweeping on/off $\quad(4 \mathrm{~s} / 2 \mathrm{~s})$; $P=600 \mathrm{~W} ; t=300 \mathrm{~s})$. Melts were poured into a sand cast mold, with the staircase configuration presented in Figure 2b. The mold included three modules (M1 to M3) to change the ratio between the melt volume and heat exchanging surface area, thus promoting different cooling rates. A K-type thermocouple (Figure 2b) was placed in the M1 module section to record the temperature variation during the melt solidification using a National Instruments CompactDAQ data logger. For comparison purposes, the same procedure was repeated without ultrasonic melt treatment to obtain non-treated melt samples.

Microstructural characterization of the cast samples (Figure 2c) was performed by optical (OM - Leica DM2500M) microscopy. Preparation of the samples for metallographic analysis included the polishing with $\mathrm{SiC}$ abrasives down to

Table 1. Alloy Chemical Composition

\begin{tabular}{llllllllll}
\hline Alloy & \multicolumn{1}{l}{ Chemical composition $(\mathrm{wt} \%)$} & & & & \\
\cline { 2 - 11 } & $\mathrm{Si}$ & $\mathrm{Mg}$ & $\mathrm{Fe}$ & $\mathrm{Ti}$ & $\mathrm{Cu}$ & $\mathrm{Mn}$ & $\mathrm{Zn}$ & Res. & $\mathrm{Al}$ \\
\hline A356 & 7.44 & 0.30 & 0.13 & 0.11 & 0.07 & 0.07 & 0.05 & 0.12 & Bal. \\
\hline
\end{tabular}
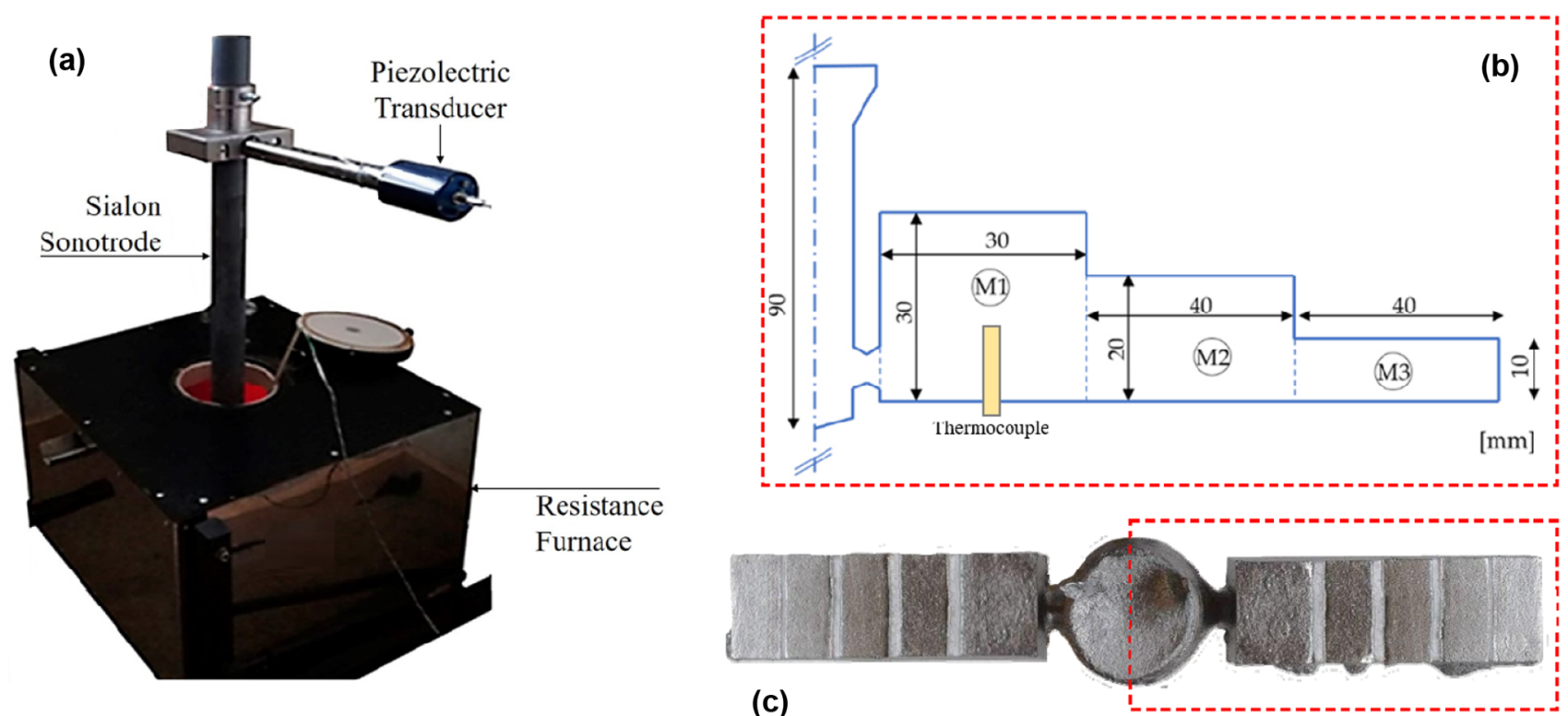

Figure 2. Experimental setup: (a) application of SiAION sonotrode in the melt, (b) mold design and (c) cast specimen. 


\section{Signal sampling and processing of output time-domain using Python programming language}

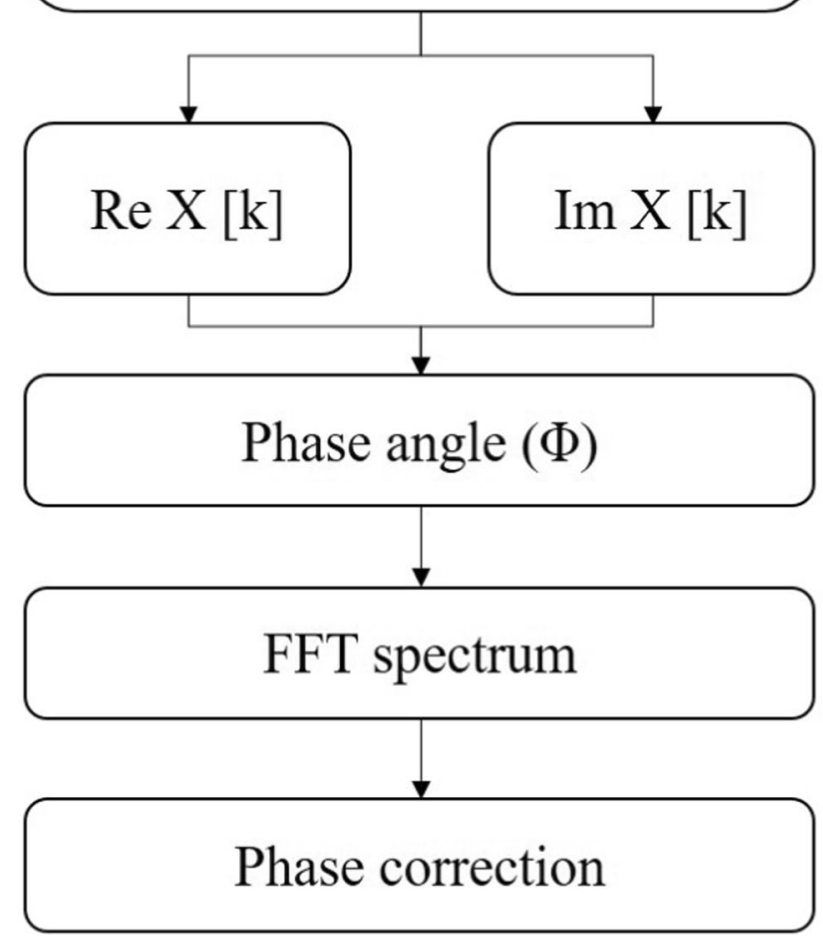

Figure 3. Flowchart on the procedure for data assembly.

a $11 \mu \mathrm{m}$ granulometry. The sample polishing was finished using $1 \mu \mathrm{m}$ diamond solution, cleaned and dried before the microscopies. The grain size was measured using the optical microscopies as reference, in accordance with ASTM-112, in ten different fields. The eutectic Si area and shape factor (particle length/width) were calculated using an algorithm that is programmed to automatically threshold and isolate these secondary phases based on color/shade identification. The same algorithm was tuned to isolate individual $\alpha$-Al grains to highlight their shape/geometry.

\section{Signal Acquisition and Analysis}

The radial vibration in the sonotrode is transmitted through elastic waves in the sonotrode surface, i.e., Lamb waves, propagating in the curved surface of the tubular structure where the tangential dimensions are much greater than the thickness. The velocity of this propagation depends on the wavelength-thickness interaction and the Lamb waves in cylindrical configurations. Fundamental resonant modes include longitudinal, torsional and flexural modes, while other resonance modes appear by the combination of these shapes and, even though, the referred modes govern wave propagation in low frequencies, tubular structures display a shell-type surface Lamb waves at high frequencies due to the low amplitudes and combination of the referred fundamental resonant modes. The sonotrode out-of-plane velocity time-domain signal of the measured points (Figure 1) was obtained experimentally using SLDV, and these vibration signals were subsequently converted into radial displacement time-domain signals using Python script subroutines.

The propagation of Lamb wave occurs by transporting energy in kinetic and potential forms, thus the time-series Lamb-wave velocity signals were assessed in accordance with relevant energy distribution in the time domain. In the present study, the discrete-time Fourier transform (DTFT) was chosen to determine the frequency content of a timedomain signal and it is used to calculate the displacement spectrum. The continuous velocity-time signal, $V(t)$ of each point was imported to the Python IDE for further signal processing. To estimate the frequency content of a given signal, a specific sampling rate $1.95 \times 10^{-5}$ was used to represent this analog continuous-time signal. To obtain the discrete sequence, the time axis was normalized to the sampling period by $T_{\mathrm{s}}=1 / f_{\mathrm{s}}$. Thus, the DTFT of an input sequence is given by Eqn. $1 .^{38,39}$

$X(f)=\sum_{n=-\infty}^{\infty} V(n) \mathrm{e}^{-j n f}$

Eqn. 1

The function $X(f)$ is used to approximate the spectrum of $V(n)$ using $2 \pi / N$ spacing between frequency points, while the $N$ is the number of samples in each $X(f)$ period. Therefore, the frequencies of the set sinusoids are presented in form as $2 k \pi / N\left(k=0,1,2, \ldots, N^{-1}\right)$, and the complex exponential basis function is determined by Eqn. 1. Eventually, the original signal $V(n)$ is obtained by estimating the weighted sum of the base functions, according to Eqn. 2.38,39

$$
\begin{aligned}
X[k] & =\sum_{k=0}^{N-1} V[n] \mathrm{e}^{-j \frac{2 \pi}{N} k n}=\sum_{k=0}^{N-1} V[n] \cdot W, \\
n & =0,1,2, \ldots, N-1
\end{aligned}
$$

Eqn. 2

where $X[k]$ represent the weight used for the complex exponential which can be equivalent to the following matrix (Eqn. 3): ${ }^{38,39}$ 


$$
\begin{aligned}
& {\left[\begin{array}{c}
X(0) \\
X(1) \\
X(2) \\
\vdots \\
X(N-1)
\end{array}\right]=\left[\begin{array}{cccccc}
1 & 1 & 1 & 1 & \ldots & 1 \\
1 & W & W^{2} & W^{3} & \ldots & W^{N-1} \\
1 & W^{2} & W^{4} & W^{6} & \ldots & W^{N-2} \\
1 & W^{3} & W^{6} & W^{9} & \ldots & W^{N-3} \\
\vdots & \vdots & \vdots & \vdots & \vdots & \vdots \\
1 & W^{N-1} & W^{N-1} & W^{N-3} & \ldots & W
\end{array}\right]} \\
& {\left[\begin{array}{c}
V(0) \\
V(1) \\
V(2) \\
\vdots \\
V(N-1)
\end{array}\right]}
\end{aligned}
$$

Eqn. 3

$N$ determines the number of the system and the values of $X[k]$ are the DTFT of the sequence output velocity-time signal. On the other hand, by decomposing Eqn. 1 into real (first term) and imaginary (second term) parts, the frequency domain of phase and amplitude for each point may be plotted. The governing equations for calculating amplitude and phase of $V(n)$ signal are given by Eqns. 4$6:^{38,39}$

$$
X[k]=\underbrace{\sum_{k=0}^{N-1} V(n) \cos \left(\frac{2 \pi k n}{N}\right)}_{\text {Real component }(\operatorname{ReX}[\mathrm{k}])}-\underbrace{i \sum_{K=0}^{N-1} V(n) \sin \left(\frac{2 \pi k n}{N}\right)}_{\text {Imaginarycomponent }=\operatorname{ImX}[\mathrm{k}]}
$$

Eqn. 4

$|X[k]|=\sqrt{\left(\operatorname{Re} X[k]^{2}\right)+\left(\operatorname{Im} X[k]^{2}\right)}$

Eqn. 5

$\Phi(\omega)=\tan ^{-1} \frac{\operatorname{Im} X[k]}{\operatorname{Re} X[k]}$

Eqn. 6

An overall description of this procedure is represented in Figure 3. Initially, the real $(\operatorname{Re} X[k])$ and imaginary (Im $X[k])$ of velocity profiles of each column of points are analyzed to calculate the individual phase angle and the FFT profile, using the referred Python script. Phase correction was performed to correct the time-shifting for phase differences on the discrete spectrum.
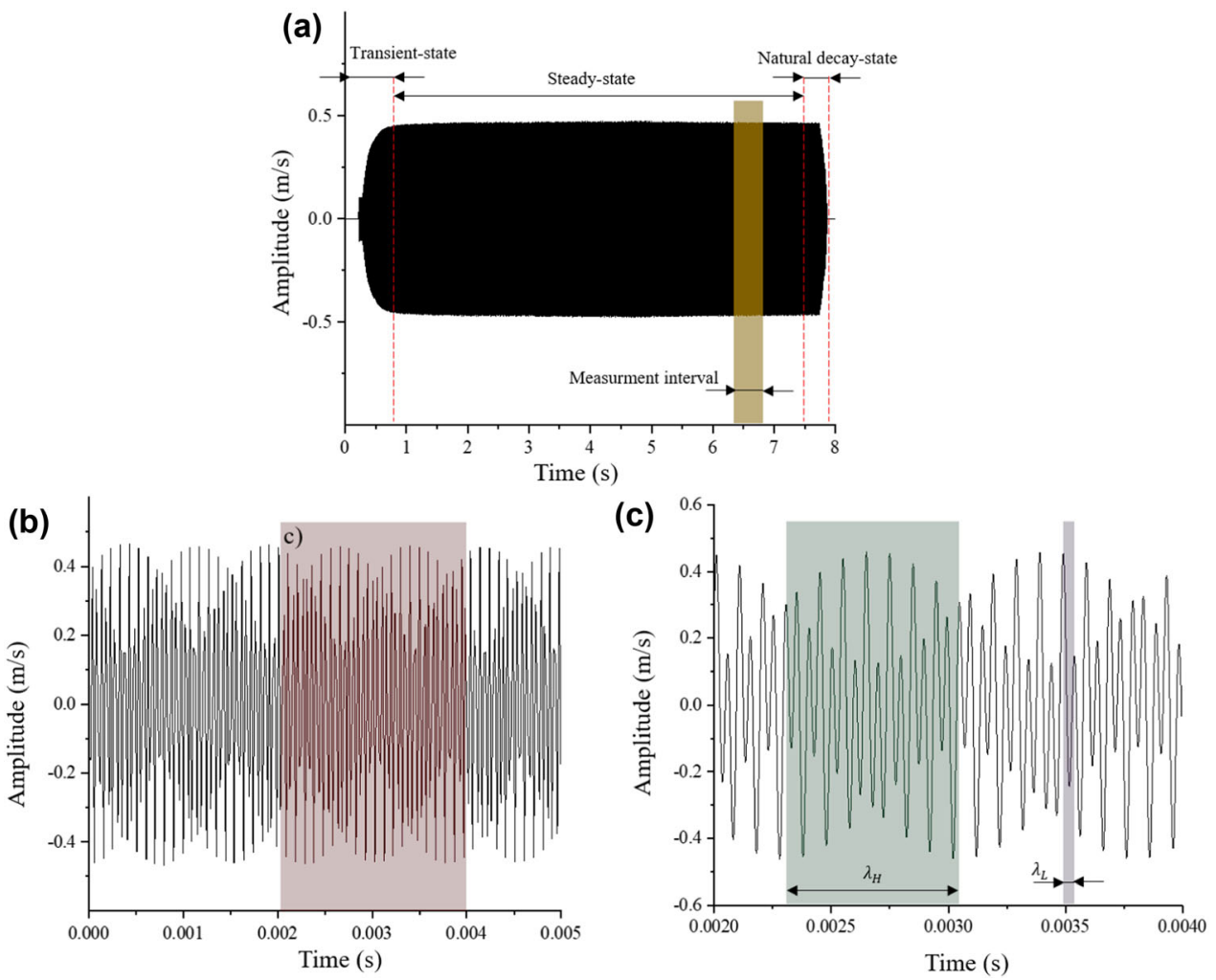

Figure 4. Experimental velocity time-domain response in Point 1: (a) full measurement; (b) time-domain responses interest interval (steady-state); and (c) refinement for modal for time-domain velocity signal. 


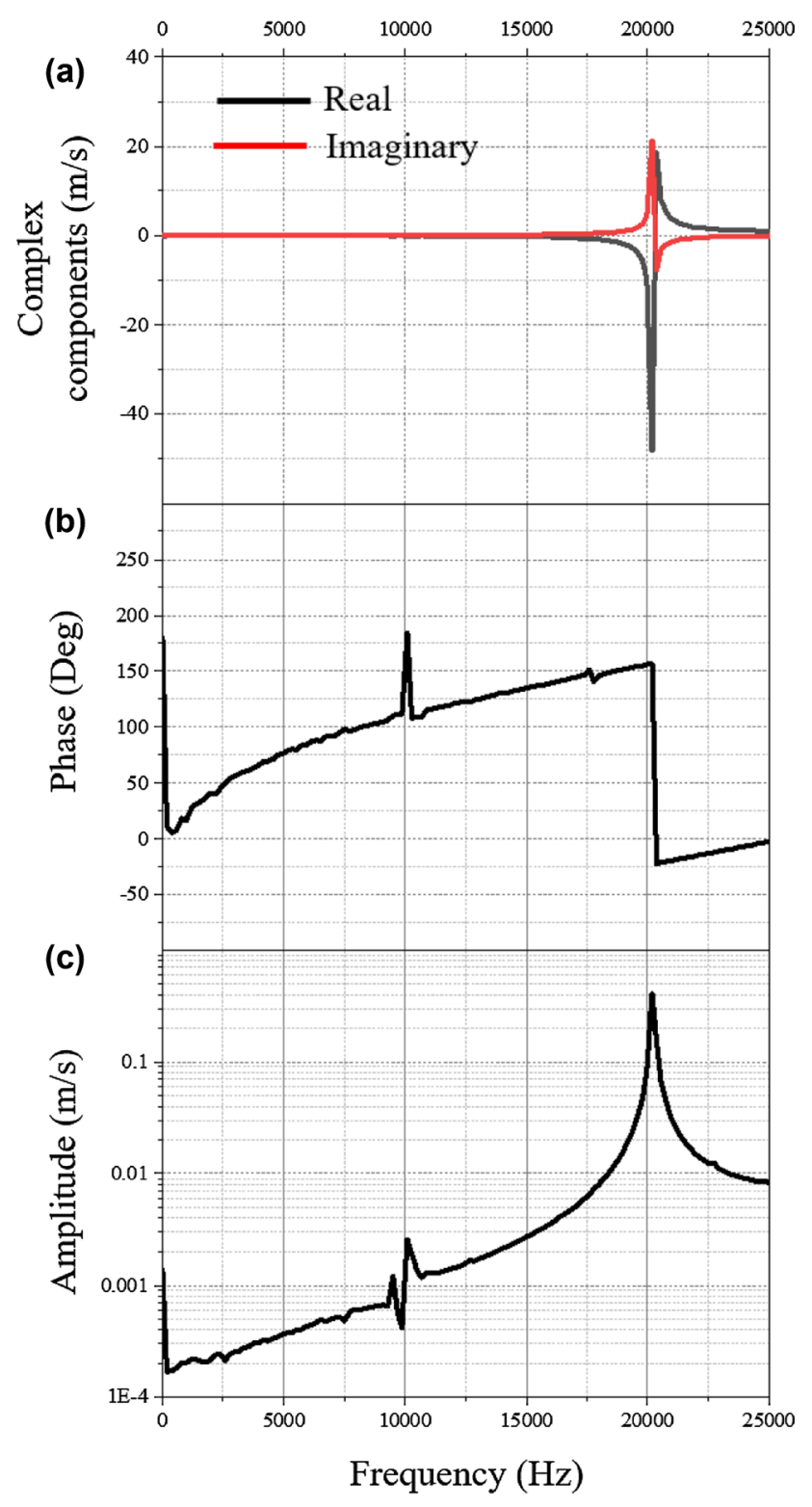

Figure 5. Fast Fourier transformation analysis and constructive frequency-domain plots of (a) real and imaginary parts; (b) phase angle; (c) velocity amplitude. (e.g., Point 1-Figure 1).

\section{Results and Discussion}

\section{Resonant Sonotrode Characterization}

Figure $4 \mathrm{a}$ shows the spectra of velocity in the time domain of a representative point (Point 1 -Figure 1) at a driving frequency of $20 \mathrm{kHz}$. Three distinct zones may be observed: transient, steady-state and natural decay states. Transient stage amplitude is increased from a stationary state toward the regular steady state after the ultrasonic excitation is switched on, where the maximal amplitudes of the waves can be kept relatively constant, and when the sonotrode vibrates in a uniform, harmonic way with stable operating frequency. The final decay state occurs when the input ultrasonic excitation is switched off, and the sonotrode itself returns to a stationary situation.

The velocity time domain was considered within the steady state during the intermodal energy exchange, to observe the dynamic response of the sonotrode (Figure 4b). It may be seen that the steady state reaches equilibrium after the initial transient stage. The measurement interval in the steady state facilitates the characterization of vibrations in terms of amplitude and phase, during the sonotrode operation, thus avoiding undesirable responses taking care only of what is relevant here. ${ }^{40}$

Figure $4 \mathrm{c}$ displays a shorter time-domain range compared to Figure $4 b$, in which the frequency response at steady state is shown to be composed of two distinct resulting $v$ shaped profiles. These may be originated from the excitation of more than a single eigenfrequency/mode. At the onset ultrasonication process, the power supply scans around $( \pm 250 \mathrm{~Hz})$ the intended eigenfrequency and avoiding the excitation of a second natural frequency. However, in steady-state regimes, the energy that is transferred along the sonotrode by the ultrasonic excitation (externally driven mode) in the tube itself may decompose by reflection and generate secondary resonances characterized by internal resonant modes. Due to these observations, it may be deduced that the signal waveforms may result in a hybrid low- and high-frequency signals, shown in Figure $4 \mathrm{c}$ as the two different $v$-shaped waveforms low $\left(\lambda_{\mathrm{L}}\right)$ and high $\left(\lambda_{\mathrm{H}}\right)$ wavelengths.

Figure 5a-c demonstrates the frequency domains of complex components, such as phase, and amplitude in the frequency domain for a representative point (Point 1Figure 1) by the application of the described method and computational routine. A clear transition in terms of complex component, phase angle and amplitude of the velocity is visible in the vicinity of $20 \mathrm{kHz}$, as this is the eigenfrequency that the system is designed to excite. However, the reflections in the steady state also promote an excitation at $10 \mathrm{kHz}$ that may be observed in Figure $5 \mathrm{~b}$ and c.

An example (Point 1-Figure 1) of the resultant conversion from the velocity time domain may be observed in Figure 6a. The areas under the velocity time-domain plot may be calculated by the partition of the total area into a small fraction trapezoid at a specific time step (e.g., $\Delta t=10^{-6} \mathrm{~s}$ ). Recurring to this method, the velocity profiles in the frequency domain, for all columnar sensors, using the Python script, were plotted in Figure 6b. It may be observed that the entire SiAlON tube has a predominant eigenfrequency in $20 \mathrm{kHz}$. Moreover, a full frequency profile of all columnar points changes the amplitude of velocity in the length of the tube, alternating between high and low amplitudes. Considering the influence of an angle, relative to the ultrasonic excitation direction, it may be 

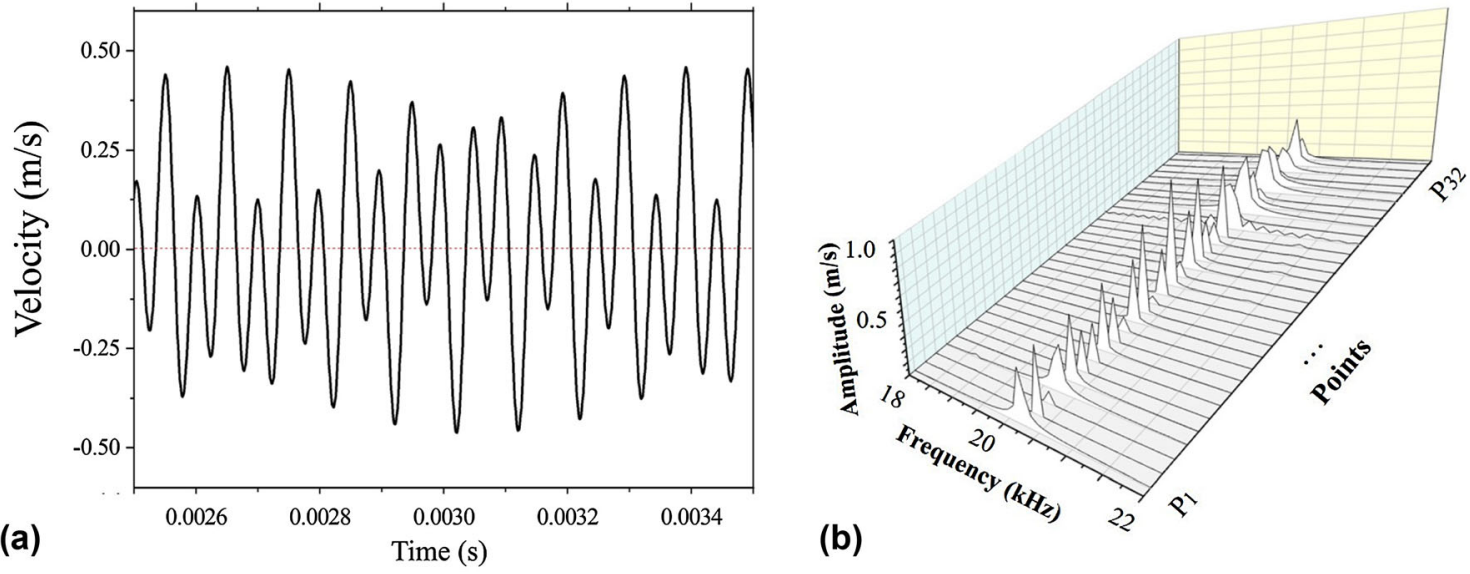

(b)

Figure 6. Results on (a) velocity in the time domain in Point 1 and (b) frequency spectrum of sonotrode columnar point.
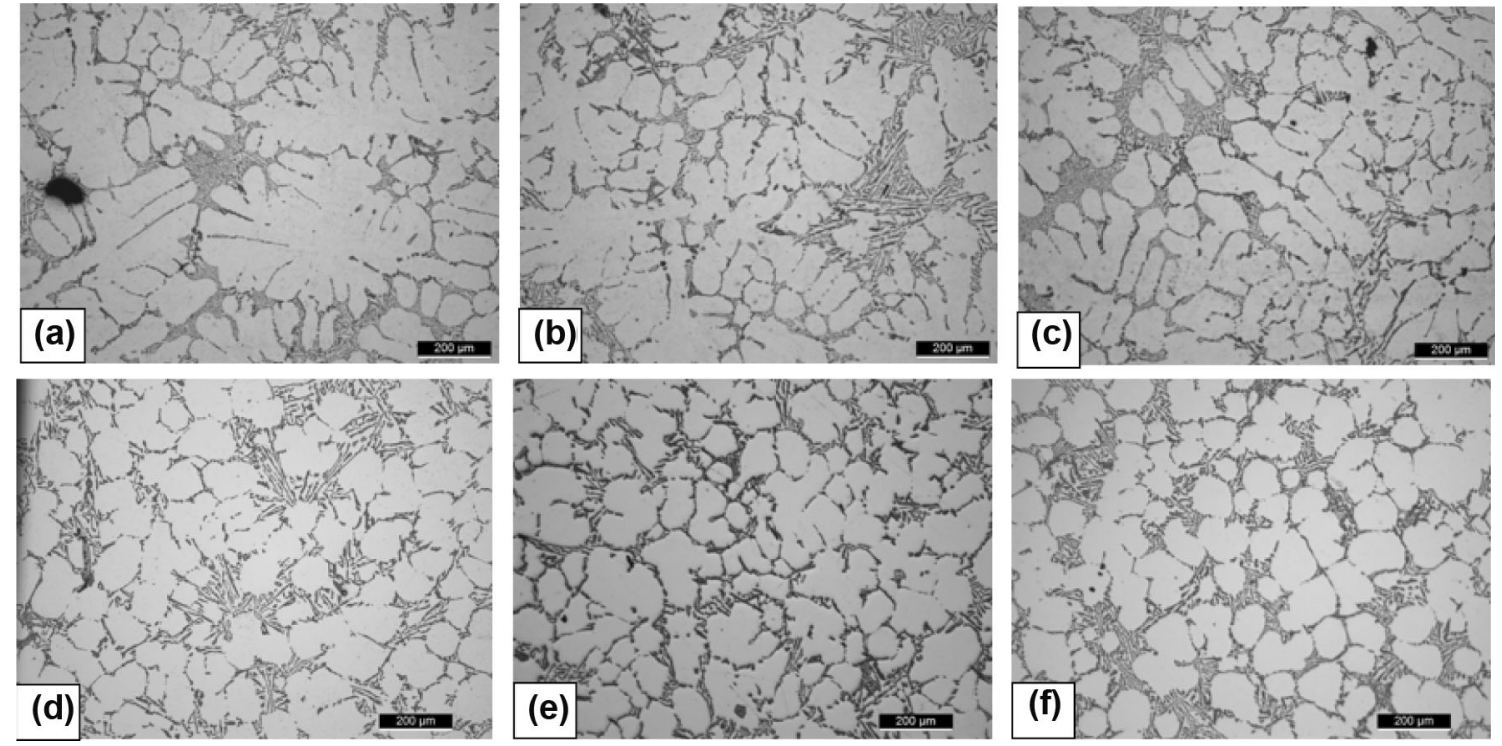

Figure 7. Microstructures in non-treated melts in the (a) M1, (b) M2 and (c) M3 modules. Microstructures in ultrasonic treated melts in the (d) M1, (e) M2 and (f) M3 modules.

observed that the overall radial distribution of velocity profiles is not axisymmetric. This is a crucial conclusion for the application of this kind of tools, recurring to the developed characterization it is showed that ultrasonic transmittance in this kind of radial tools could not be considered as equally efficient in the whole tube length.

In an ultrasonic tool perspective, it is shown that the efficiency of the radial ultrasonic transmission may not uniform in the used medium, as it depends in the sonotrode cartesian (i.e., length) and polar (i.e., angular) directions. In the particular light alloy melt treatment, grain refinement and degassing depend on the generation of cavitation in the fluid medium. As ultrasonic melt treatment promotes local static pressure of the fluid to reach a level below vapor pressure, the acoustic-activated medium near the sonotrode creates and disperses cavitation. However, the performed characterization proves that this technique is able to promote cavitation in larger volumes given its influence in the overall surface area, relative to tip-activated ultrasonic sonotrodes. $^{41}$

\section{Melt Treatment Characterization}

To evaluate the efficiency and performance of the designed ultrasonic system, the samples were cast with and without being excited by the ceramic sonotrode. While it is expected that the alloy bath lowers the natural frequency (reduction in elastic modulus with temperature), the resonant amplitudes themselves are not changed. Even though the system damping increases (i.e., increase in loss modulus and sonotrode/melt friction), the MMM technology is able to compensate these factors and output the same amplitude that is observed in the air. Thus, the surface characterization using the air approach is directly 

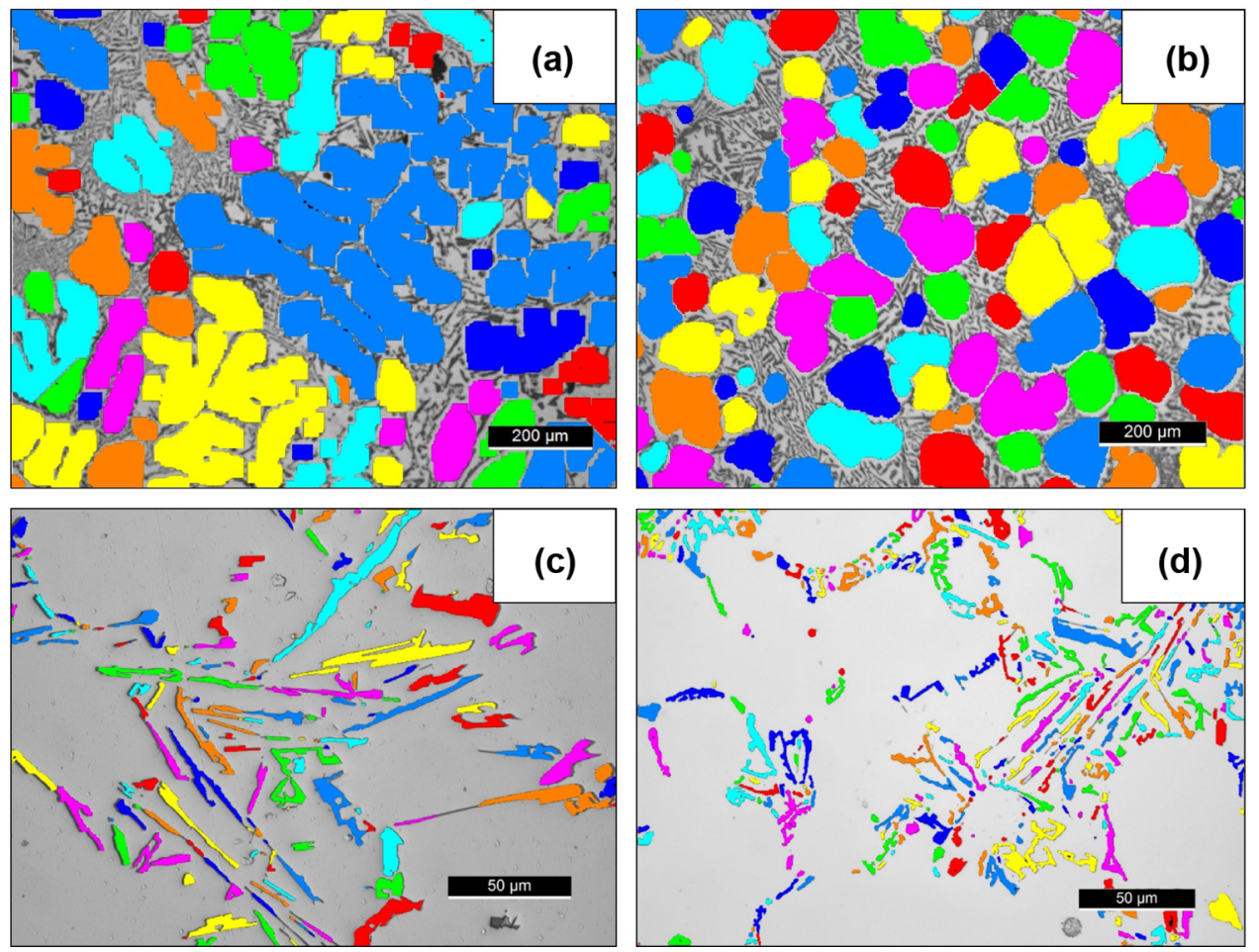

Figure 8. Microstructural details by thresholding algorithm in M3 module: $\alpha-A I$ grains in (a) non-treated and (b) US treated melts; Eutectic Si in (c) non-treated and (d) US treated melts.

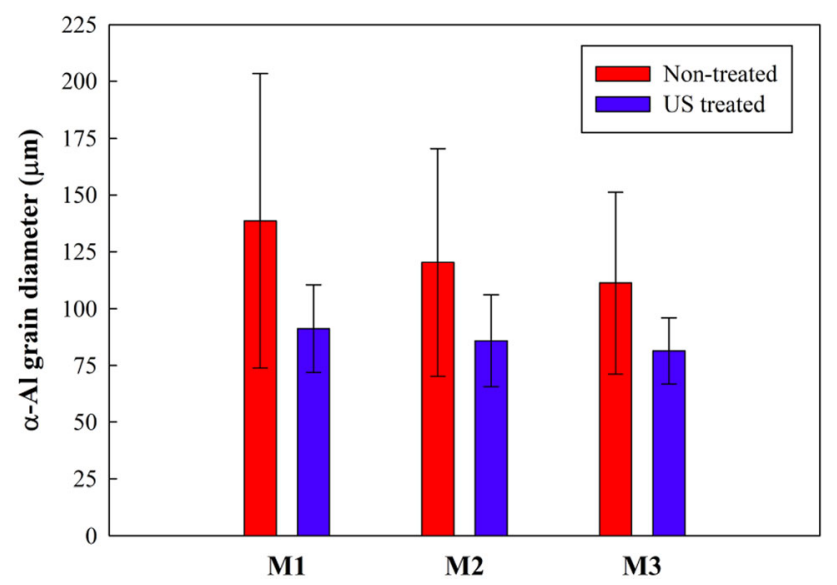

Figure 9. Characterization of mean $\alpha-A l$ grain size.

correlated with its behavior inside the melt. The resultant microstructures corresponding to non- and ultrasonically treated samples may be observed in Figure 7, where it is shown that the ultrasonic melt treatment has a profound effect on the overall grain and secondary refinement. This effect seems to be influenced by the changes in the solidification rate that is promoted by different modules (M1, M2 and M3-Figure 2b).

Comparing the non-treated melts (Figure $7 \mathrm{a}-\mathrm{c}$ ) with those treated by ultrasound (Figure 7d-f), it is suggested that the Lamb waves in the surface of the sonotrode are able to promote an excitation in the melt that is observed up to the last module (3). Figure 8a shows that untreated melts promote dendritic $\alpha$-Al grain morphology, hypothesizing that this processing condition has a lower cooling rate. However, samples with ultrasonically treated melts (e.g., Figure 8b) show a transition into a heterogeneous solidification promoted by the alternating pressure. The latter introduces cavitation in the melt, since it is well established that the collapse of cavitation bubbles promotes heterogeneous nucleation due to local pressure changes. ${ }^{7}$ Thus, as a grain germinator, US is able to stimulate the nucleation of a high fraction of globular grains. ${ }^{21}$

This is further validated by Figure 9, which illustrates that by changing the modules (i.e., modifying the thermal conductivity with the mold), different cooling rates have a higher impact on the samples with non-treated melts. In the samples with ultrasonically treated melts, the value of the grain diameter remains fairly stable in different modules, i.e., the grain size is more dependent on the rate of heterogeneous nucleation due to cavitation (i.e., intrinsic thermodynamic balance within the proto-grains) than due to grain growth by heat exchange.

Figure 10 shows that the precipitation of proeutectic and eutectic $\alpha$-Al is initiated at a higher temperature in the samples with US treated melts, resulting in an elevation of the solidification rate and reduction in the solidification time. Also, the hypothesis that non-treated melts have lower cooling rates is corroborated. According to 

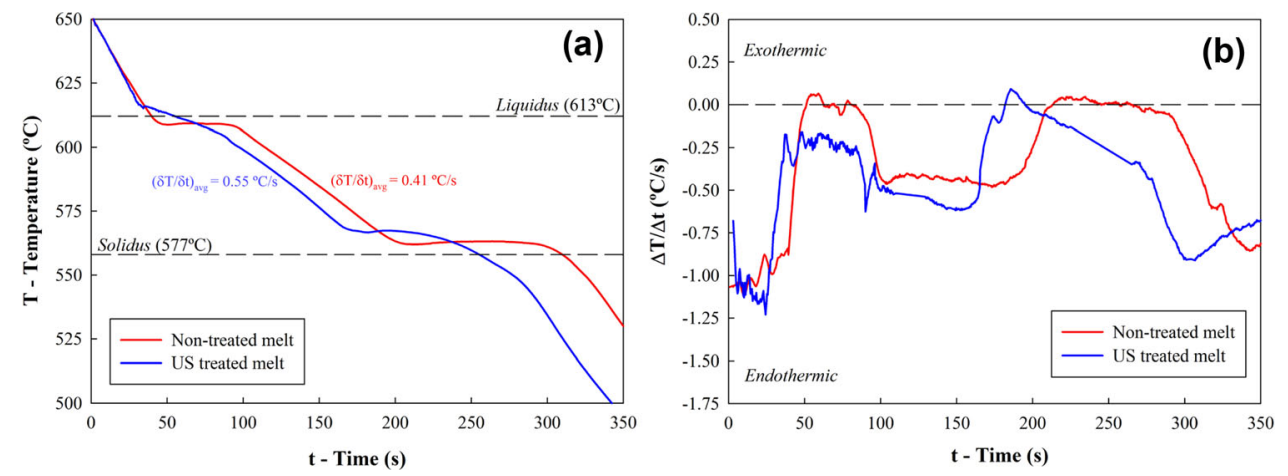

Figure 10. Solidification in M1 module: (a) cooling curves and (b) first derivative.
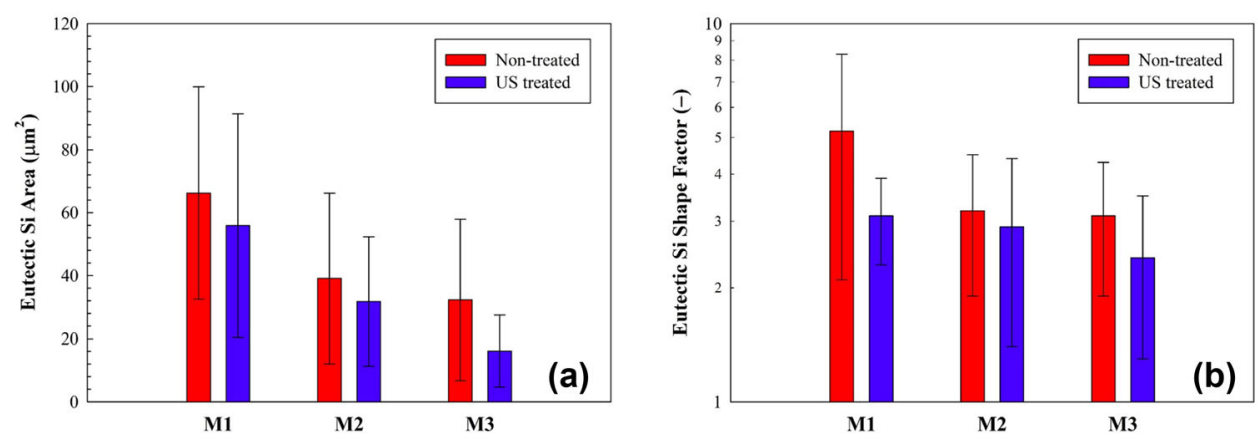

Figure 11. Characterization of eutectic Si: (a) area and (b) shape factor.

Figure $10 \mathrm{~b}$, due to the presence of exothermic reactions during the liquidus transition in the solidification of the non-treated samples, it is expected that dendrites are nucleated. This is, in fact, observable in Figures 7 and 8.

In relation to the eutectic $\mathrm{Si}$, the comparison between Figure $8 \mathrm{c}$ and $\mathrm{d}$ suggests that ultrasonic melt treatment is an effective technique for modification and refinement, as confirmed by the data in Figure 10. However, Figure 11a shows that the cooling rate is a relevant variable in the overall area of the eutectic Si. Given that the intergranular space is approximate (grain size does not change significantly, according to Figure 9) and the modification of eutectic Si by acoustic energy is related to its fracture spheroidization by cavitation followed by a higher solidification rate, the change in area in Figure 10a must be related to the higher cooling rates in section M3.

This is further confirmed by the tendency to lower the of shape factor (length $\times$ thickness, Figure 11b) of the eutectic $\mathrm{Si}$ in samples with US treated melts. While the lowering of the shape factor in non-treated samples may be attributed by the reduction of intergranular space in the different modules (Figure 9), in samples with US treated melts and due to the lower variation in grain size, this is suggested to be correlated with the cavitation that is promoted by the acoustic energy.

\section{Conclusions}

This study reports the design of a script and visualization routine for the characterization of the dynamic behavior of ultrasonic tools with complex shapes and low-amplitudes. An example of a tubular sialon sonotrode used for lightalloy melt treatment is analyzed to validate the developed routine, being the following conclusions drawn:

- The Python-based algorithm is able to interpret the input signal and determine the time-domain velocity profiles of the different measurement points to allow a characterization of the sonotrode radial vibration. Measurement points could be correlated in the velocity time domains, considering different positions;

- Data assembly was successfully performed, being shown that the vibration response is dependent on the distance of ultrasonic excitation incidence;

- Radial resonant natural modes display a distributed ultrasonic vibration, and consequently cavitation, in the surface of the sonotrode. Thus, the refinement of light alloys by the described technique is proposed to more appropriate for larger medium and/or continuous mediums;

- Lamb waves have been shown as the main mechanism to promote ultrasonic excitation in melts using tubular ceramic sonotrodes and are 
able to promote $\alpha$-Al grain refinement and modification of the eutectic $\mathrm{Si}$;

- It is shown that the alloy refinement and modification in US treated melts is more dependent on the cavitation promoted by acoustic excitation than by the effect of cooling rate, either by heterogeneous $\alpha$-Al grain nucleation or fragmentation/spheroidization of the eutectic Si.

\section{Acknowledgments}

This work was supported by PTDC/EMEEME/30967/ 2017 and NORTE-0145-FEDER-030967, co-financed by the European Regional Development Fund (ERDF), through the Operational Programme for Competitiveness and Internationalization (COMPETE 2020), under Portugal 2020, and by the Fundação para a Ciência e a Tecnologia - FCT I.P. national funds. Also, this work was supported by Portuguese FCT, under the reference project UIDB/04436/2020, and Stimulus of Scientific Employment Application CEECIND/03991/2017.

\section{Data Availability}

The raw/processed data required to reproduce these findings cannot be shared at this time due to legal or ethical reasons.

\section{REFERENCES}

1. C.-W. Lin, C.-C. Chang, H.-T. Chen, Y.-J. Chen, C.-S. Lin, H.-C. Hsu, H.-K. Tsou, 3D real-time imageguided navigation spine corpectomy with ultrasonic bone cutter: technical note. World Neurosurg. 135, 197-204 (2020)

2. F. Chen, M. Zhang, C. Yang, Application of ultrasound technology in processing of ready-to-eat fresh food: a review. Ultrason. Sonochem. 63, 104953 (2020)

3. H. Zhou, J. Zhang, D. Yu, P. Feng, Z. Wu, W. Cai, Advances in rotary ultrasonic machining system for hard and brittle materials. Adv. Mech. Eng. 11, 1687814019895929 (2019)

4. M.A. Nunes, A.S.G. Costa, S. Bessada, J. Santos, H. Puga, R.C. Alves, V. Freitas, M.B.P.P. Oliveira, Olive pomace as a valuable source of bioactive compounds: a study regarding its lipid- and water-soluble components. Sci. Total Environ. 644, 229-236 (2018)

5. F. Marangi, D. Pinto, L. Francisco, R.C. Alves, H. Puga, S. Sut, S. Dall'Acqua, F. Rodrigues, M.B.P.P. Oliveira, Hardy kiwi leaves extracted by multifrequency multimode modulated technology: a sustainable and promising by-product for industry. Food Res. Int. 112, 184-191 (2018)

6. H. Puga, R.C. Alves, A.S. Costa, A.F. Vinha, M.B.P.P. Oliveira, Multi-frequency multimode modulated technology as a clean, fast, and sustainable process to recover antioxidants from a coffee by-product.

J. Clean. Prod. 168, 14-21 (2017)

7. D.G. Eskin, I. Tzanakis, F. Wang, G.S.B. Lebon, T. Subroto, K. Pericleous, J. Mi, Fundamental studies of ultrasonic melt processing. Ultrason. Sonochem. 168, 455-467 (2019)

8. N.Q. Tuan, H. Puga, J. Barbosa, A.M.P. Pinto, Grain refinement of $\mathrm{Al}-\mathrm{Mg}-\mathrm{Sc}$ alloy by ultrasonic treatment. Met. Mater. Int. 21, 72-78 (2015)

9. H. Puga, J. Barbosa, N.Q. Tuan, F. Silva, Effect of ultrasonic degassing on performance of Al-based components. Trans. Nonferrous Met. Soc. China 24, 3459-3464 (2014)

10. W. Khalifa, S. El-Hadad, Ultrasonication effects on the microstructure characteristics of the A380 die cast alloy. Int. J. Metalcast. 13, 865-879 (2019)

11. X. Jian, C. Xu, T. Meek, Q. Han, Effect of ultrasonic vibration on the solidification structure of A356 alloy. Trans. Am. Found. Soc. 113(131-138), 312-316 (2005)

12. P. Lynch, R. Voigt, J. Furness Jr., D. Paulsen, The effects of non-contact acoustic stimulation on the solidification behavior and microstructure of aluminum alloy A356. AFS Trans. 118, 57 (2010)

13. Y. Yao, Y. Pan, S. Liu, Power ultrasound and its applications: a state-of-the-art review. Ultrason. Sonochem. 62, 104722 (2019)

14. J. Gao, H. Caliskan, Y. Altintas, Sensorless control of a three-degree-of-freedom ultrasonic vibration tool holder. Precis. Eng. 58, 47-56 (2019)

15. C. Ma, Z. Wang, Experimental and numerical investigation of the breakage of a cutting tool with ultrasonic vibration. Precis. Eng. 51, 393-402 (2018)

16. S.-B. Kim, Y.-H. Cho, M.-S. Jo, J.-G. Jung, Y.-K. Lee, J.-M. Lee, Quantitative approach to realization of ultrasonic grain refinement of $\mathrm{Al}-7 \mathrm{Si}-2 \mathrm{Cu}-1 \mathrm{Mg}$ alloy. Sci. Rep. 9, 17812 (2019)

17. H. Puga, J. Barbosa, V.H. Carneiro, The role of acoustic pressure during solidification of AlSi7Mg alloy in sand mold casting. Metals 9, 490 (2019)

18. H. Puga, V.H. Carneiro, J. Barbosa, D. Soares, Effect of grain and secondary phase morphologies in the mechanical and damping behavior of Al7075 alloys. Met. Mater. Int. 22, 863-871 (2016)

19. C. Allen, Q. Han, Grain refinement of pure aluminum using ultrasonics. Int. J. Metalcast. 5, 69-70 (2011)

20. D.G. Eskin, Overview of ultrasonic degassing development, in Light metals 2017, ed. by A.P. Ratvik (Springer, Berlin, 2017), pp. 1437-1443

21. J. Barbosa, H. Puga, Ultrasonic melt processing in the low pressure investment casting of $\mathrm{Al}$ alloys. J. Mater. Process. Technol. 244, 150-156 (2017)

22. X. Liu, S. Jia, L. Nastac, Ultrasonic cavitation-assisted molten metal processing of cast A356-nanocomposites. Int. J. Metalcast. 8, 51-58 (2014)

23. G. Cao, H. Konishi, X. Li, Recent developments on ultrasonic cavitation based solidification processing of 
bulk magnesium nanocomposites. Int. J. Metalcast. 2, 57-65 (2008)

24. X. Li, Y. Yang, D. Weiss, Ultrasonic cavitation based dispersion of nanoparticles in aluminum melts for solidification processing of bulk aluminum matrix nano-composite: theoretical study, fabrication and characterization. AFS Trans. 133, 1-12 (2007)

25. S. Wetzel, Nano's Frontier-If you think tiny particles making metals super strong sounds like sci-fi, think again. Mod. Cast. 100, 27 (2010)

26. H. Choi, W. Cho, X.C. Li, Scale-up ultrasonic processing system for batch production of metallic nanocomposites. AFS Trans. 121, 146-151 (2013)

27. W. Cho, X.C. Li, Semi-solid mixing for fabrication of A206/ $/ \mathrm{Al}_{2} \mathrm{O}_{3}$ master nanocomposites. AFS Trans. 121, 159-164 (2013)

28. Y. Sun, H. Choi, X.C. Li, Composition optimization for $\mathrm{A} 206 / \mathrm{Al}_{2} \mathrm{O}_{3}$ nanocomposite. AFS Trans. 121, 205-215 (2013)

29. J. Barbosa, H. Puga, Ultrasonic melt treatment of light alloys. Int. J. Metalcast. 13, 180-189 (2019)

30. H. Puga, V.H. Carneiro, J. Barbosa, V. Vieira, Effect of ultrasonic treatment in the static and dynamic mechanical behavior of AZ91D Mg alloy. Metals 5, 2210-2221 (2015)

31. H. Puga, J. Barbosa, J.M. Machado, C. Vilarinho, Ultrasonic grain refinement of die cast copper alloys. J. Mater. Process. Technol. 263, 336-342 (2019)

32. X. Zhang, J. Kang, S. Wang, J. Ma, T. Huang, The effect of ultrasonic processing on solidification microstructure and heat transfer in stainless steel melt. Ultrason. Sonochem. 27, 307-315 (2015)

33. B.V. Djambazov, B. Lebon, K. Pericleous, Contactless acoustic wave generation in a melt by electromagnetic induction, in Light metals 2017, ed. by J. Grandfield (Springer, Cham, 2014), pp. 1379-1382

34. G.I. Eskin, Ultrasonic treatment of light alloy melts (CRC Press, Boca Raton, 1998)

35. T. Zeng, Y. Zhou, Effects of ultrasonic introduced by L-shaped ceramic sonotrodes on microstructure and macro-segregation of 15t AA2219 aluminum alloy ingot. Materials 12, 3162 (2019)

36. X. Chen, J. Li, Noise reduction for ultrasonic Lamb wave signals by empirical mode decomposition and wavelet transform. J. Vibroeng. 15, 1157-1165 (2013)

37. S. Huang, Z. Wei, W. Zhao, S. Wang, A new omnidirectional EMAT for ultrasonic Lamb wave tomography imaging of metallic plate defects. Sensors 14, 3458-3476 (2014)

38. A.V. Oppenheim, A.S. Willsky, S.H. Nawab, Signals \& systems (Prentice Hall, Upper Saddle River, 1983)

39. K. Shin, J. Hammond, Fundamentals of signal processing for sound and vibration engineers (Wiley, New York, 2008)

40. A. Albareda, R. Perez, J.A. Casals, J.E. Garcia, D.A. Ochoa, Optimization of elastic nonlinear behavior measurements of ceramic piezoelectric resonators with burst excitation. IEEE Trans. Ultrason. Ferroelectr. Freq. Control 54, 2175-2188 (2007)

41. I. Tzanakis, G.S.B. Lebon, D.G. Eskin, K. Pericleous, Investigation of the factors influencing cavitation intensity during the ultrasonic treatment of molten aluminium. Mater. Des. 90, 979-983 (2016)

Publisher's Note Springer Nature remains neutral with regard to jurisdictional claims in published maps and institutional affiliations. 